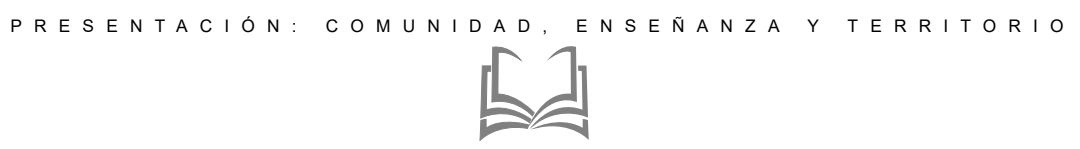

PRESENTACIÓN. REVISTA BOLETÍN REDIPE: 9 (7) JULIO 2020 ISSN 2256-1536

\title{
COMUNIDAD, ENSEÑANZA Y TERRITORIO
}

\section{COMMUNITY, TEACHING AND TERRITORY}

\author{
David Fragoso Francoํㅜ, Unam, México
}

No parecen ser suficientes ni contundentes los esfuerzos que dentro del ámbito educativo y la sociedad se adelantan en la tarea ética de restituir a la comunidad y a la tierra su valor vital, como elementos basales de la evolución de la vida. Uno y otro han venido siendo erosionados, sometidos, como la misma educación, a la lógica del progreso, del poder financiero, de una lógica que le apuesta más al mercado que a la vida, al utilitarismo que a la solidaridad, al individualismo que a las relaciones de alteridad ética como forma de mantener el orbe humano, el orbe de la vida y el planeta. Es esta la idea nuclear del número actual de nuestra Revista Boletín Redipe.

Los artículos que conforman esta edición constituyen producciones derivadas de procesos investigaciones y de indagaciones frente a los temas comunitarios, territoriales, educativos y ambientales. Veamos.

\section{COMUNIDAD, PEDAGOGÍAY SUBJETIVIDAD.}

Artículo derivado de investigación elaborado por la académica colombiana Maria Fernanda Gil Claros, Grupo de Investigación Redipe. Pone de manifiesto que comprender el concepto de comunidad en la complejidad de las realidades globales implica abrirse a horizontes en los cuales la vida, y específicamente las formas de vivir, están en entredicho por discursos epistemológicos que no han sabido recobrar la fuerza inmanente de la voluntad de vivir en los escenarios vitales, como la comunidad. De este modo la comunidad es repensada en los ámbitos de la vida por el encuentro de subjetividades no propietarias, noviolentas de la existencia, que al exponerse a un afuera saben proteger la potencia de existir. Es en este contexto que la pedagogía crítica, trasladada a lo experiencial, dispone su en Ciencias Humanas y Doctorando en Comunicación y Pensamiento Estratégico. Profesor de Carrera Titular en el área de Comunicación Educativa en el Programa de Pedagogía de la Facultad de Estudios Superiores Acatlán de la Universidad Nacional Autónoma de México. Ha publicado diferentes artículos de libros, textos académicos y artículos para publicaciones periódicas. Ha participado en diferentes órganos colegiados de decisión en la UNAM. Sus líneas de investigación son: comunicación en el aula, educación para los medios, cognición y afectividad en la didáctica. Proyectos integradores interdisciplinarios en Educación Superior. David Fragoso Franco (0000-0003-3277-2841) 
lugar en el pensamiento vivo, comprometido con saberes que, como tékhne, construyen lo posible desde las acciones coherentes de una palabra no explicadora.

\section{CUIDAR Y CRIAR EN COMUNIDADES} ORIGINARIAS: UNA APUESTA DE INVESTIGACIÓN INTERCULTURAL ENTRE COLOMBIA-BRASIL. Artículo de investigación de los académicos Lorena Cardona Alarcón, Fundación Universitaria del Área Andina, Bogotá- Colombia- Eliane Rodrigues de Castro- Rede Pública Municipal de Juiz de Fora/ UFJF, (Minas Gerais)- Brasil. Producto del intercambio académico y el diálogo intercultural entre investigadores de Brasil y Colombia, en el marco del proyecto Geografías del cuidado y la crianza desde la experiencia educativa en comunidades originarias de América Latina, una apuesta que adelantan la Fundación Universitaria del Área Andina (Colombia) y la Universidad Federal Juiz de Fora (Brasil), a través de sus Facultades de Educación y los grupos de investigación Kompetenz y GRUPEGI, respectivamente, como respuesta a los retos que los contextos socioculturales colombianos, brasileños y latinoamericanos, le demandan a la academia desde los saberes originarios que necesitan ser visibilizados para comprender las realidades diversas de nuestros niños y niñas.

\section{¿PORQUÉLAFRONTERASOCIOAMBIENTAL ES UN CONCEPTO IMPORTANTE EN LOS ESTUDIOS TERRITORIALES?* Artículo de investigación autoría de los académicos Juan} Manuel Ochoa Amaya, Universidad de los Llanos, Luís Llanos Hernández- Universidad Autónoma de Chapingo. Identifica la relación de los seres humanos con un modelo económico dominante, que deja trazos indelebles sobre el espacio, estudiados con la finalidad de entrever nuevos paradigmas y, llegado el caso, plantear conceptos para estudiar el territorio. Así, se aborda esta problemática tomándose como punto de partida el desarrollismo económico, el cual es tomado como el origen de la crisis ambiental por la cual atraviesan los territorios. De otra parte, se analiza cómo a través de la conciencia de los seres humanos, es decir, a través de la capacidad que tienen para restañar o restablecer, éstos se pronuncian por la defensa o cuidado de la naturaleza, adoptando una posición "conservacionista" que la pretende mantener inmaculada. En las últimas décadas ha sido el eje de la política internacional de tipo ambiental. Lo anterior devela que contrario al criterio clásico de racionalidad económica, la racionalidad ambiental es una postura que busca mantener el equilibrio de la naturaleza con la humanidad. El desarrollismo impulsado por la racionalidad económica ha colocado al mundo en una condición incierta. En el artículo también se propone el concepto de frontera socioambiental como un recurso epistemológico para el estudio del territorio en su vertiente ambiental.

\section{CONCEPTODELAGUAYSUSIMPLICACIONES EN LA FORMACIÓN AMBIENTAL Artículo de} investigación presentado por las académicas colombianas Diana Marcela Trujillo SuárezLiliana Caycedo Lozano Unicolmayor, Colombia. Aseguran que el cuidado de las fuentes hídricas en el territorio colombiano es una prioridad que se enmarca dentro de los desafíos que surgen a partir de la propuesta de los Objetivos de Desarrollo Sostenible. En este contexto, es importante el papel que juega la Educación Ambiental en todos los niveles de formación. El trabajo reflexiona en torno a los resultados de dos investigaciones llevadas a cabo por el grupo de Investigación Planificación en Gestión Ambiental Eficiente de la Universidad Colegio Mayor de Cundinamarca que evidencian la necesidad de la fundamentación conceptual para trascender con argumentos, desde la valoración del agua solo como recurso hídrico hasta la valoración del compuesto como un reactivo con propiedades definitivas para la sostenibilidad del planeta y de nuestro territorio. 
EDUCACIÓN INCLUSIVA EN LAS INSTITUCIONES DE EDUCACIÓN SUPERIOR QUE OFERTAN PROGRAMAS POR CICLOS PROPEDÉUTICOS EN BOGOTÁ 2010 - 2020: REVISIÓN DE LA LITERATURA. Artículo de revisión elaborado por la académica colombiana Cecilia Garzón Daza- Fundación Universitaria San Mateo, Universidad Católica de Colombia. Indaga el estado del arte de los avances de la educación inclusiva en las Instituciones de Educación Superior que ofrecen programas por ciclos propedéuticos en Bogotá 2010 - 2020. Desde este punto de vista la inclusión se ha constituido en el estandarte de una educación más humana, que busca integrar la diferencia, sin importar el carácter que esta tenga; bien sea que esta obedezca a aspectos ideológicos, religiosos, raza, orientación sexual, entre otros; para tal fin el artículo se estructura en tres apartados, el primero orientado a contextualizar las Instituciones de Educación Superior que se orientan por ciclos propedéuticos según la Ley 749 de 2002, el segundo identificar los avances en materia de inclusión educativa en el país; el tercero la revisión del estado del arte, análisis de los avances y retrocesos en la materia.

\section{TRANSFORMACIÓN DE LA EDUCACION FRENTE A LA PANDEMIA Y LA ANALITICA} DE DATOS. Artículo de investigación a cargo de los académicos Leonardo Emiro Contreras Bravo Héctor Javier Fuentes López Karolina González Guerrero. Se presenta un panorama de los cambios que está sufriendo el sector educativo de manera acelerada por la adopción de tecnologías y plataformas para apoyar un proceso que tradicionalmente era presencial. Esta puede ser una oportunidad para aprovechar la información disponible de los procesos educativos y mediante el uso de técnicas como el machine learning, obtener conocimiento que permita la toma de decisiones oportunas alrededor de las problemáticas que aquejan a las Instituciones de educación. Debido a la emergencia de salud ocasionada por el
COVID-19 las comunidades académicas se han visto afectadas en su salud mental evidenciando factores de ansiedad y frustración ante la nueva realidad.

\section{LA INTERVENCIÓN PEDAGÓgICA EN} EDUCACIÓN FíSICA. Artículo de investigación a cargo los académicos Eulisis Smith Palacio y Jesús Alcalá Recuerdo, Universidad Francisco de Vitoria. España. Analiza las diferencias por curso en Educación Primaria Obligatoria, en la adquisición del autocontrol en preadolescentes que participaron del Programa de Intervención Educativa Delfos, Cecchini (2008). Se confirma una disminución significativa en cuanto a las protestas, la violación de las reglas del juego, al igual que la opinión sobre la búsqueda de la victoria a cualquier precio, resaltando valores prosociales, respeto a los compañeros y respeto a las normas de juego.

\section{UNA APROXIMACIÓN A LAS CARACTERÍSTICAS DE DOCENTES TALENTOSOS EN EDUCACIÓN SUPERIOR.} Artículo de investigación elaborado por los académicos Diana Irasema Cervantes Arreola, Alejandro Jesús Robles Ramírez, Fabiola Lom Monárrez, Universidad Autónoma de Ciudad Juárez- Erick Ríos Díaz, Centro Educativo Hidalgo, México. Muestra un acercamiento a las características atribuidas de los estudiantes a los docentes talentosos en educación superior. Los resultados ponen de presente que las características más atribuidas son: el dominio de contenidos, ser creativo, respetuoso, con comunicación asertiva, que promueve el desarrollo de sus estudiantes y brinda una explicación clara. Las relaciones que puntúan de manera más significativa con sexo y nivel que cursa el estudiante, son: ser responsable, dar acompañamiento en casos de rezago y el ser comprensivo. 
METODOLOGÍA POLYA EN EL DESARROLLO DE LA COMPETENCIA INTERPRETACIÓN EN LA JUVENTUD. Artículo de investigación elaborado por los académicos Jhon Jairo Meneses Patiño - Mawency Vergel OrtegaJhan Piero Rojas Suarez Facultad de Ingeniería, Universidad Francisco de Paula Santander, Colombia. Trabajo asociado al diseño e implementación de una estrategia pedagógica para el desarrollo de la competencia interpretación y resolución de problemas matemáticos basada en la metodología de Polya. El método empleado para abordar el problema fue el de Solomon. El trabajo se desarrolla mediante la implementación de guías, que debieron resolverse de forma individual. Los resultados mostraron que la implementación de la metodología de Polya contribuye a la construcción de estructuras mentales que facilitan el proceso de resolución de problemas, especialmente en lo concerniente a la interpretación del problema y a la verificación de los resultados obtenidosx.

\section{LA INCORPORACIÓN DE LAS TIC EN EL DESARROLLO HISTÓRICO DE UNA LICENCIATURA EN EDUCACIÓN. Artículo de revisión elaborado por los académicos}

\section{Karla Lariza Parra Encinas- Arnoldo} Lizárraga Juárez, Universidad Autónoma de Baja California. Presenta una revisión histórica de las diversas funciones que han cumplido las TIC a lo largo del desarrollo de la Licenciatura en Ciencias de la Educación de la Universidad Autónoma de Baja California (UABC), desde sus inicios hasta la creación del Centro de Educación Abierta y a Distancia. La revisión fue realizada con base en una investigación documental de los archivos de la UABC en general y de la Facultad de Ciencias Humanas en lo particular, y se enmarca en los contextos institucional y nacional a través del modelo educativo de la UABC, los Planes Nacionales de Desarrollo de México y los Planes Sectoriales de Educación.
INFLUENCIA DE LOS RECURSOS DIGITALES EN LA ENSEÑANZA $Y$ EL APRENDIZAJE DEL IDIOMA INGLÉS EN LAS ESCUELAS NORMALES DE OAXACA, MÉXICO. Artículo de investigación elaborado por los académicos Karen Josefa González Vega - Universidad La Salle, Oaxaca, México. Orientado a determinar la influencia que tienen los recursos digitales en la enseñanza y aprendizaje del idioma Inglés en las Escuelas Normales de Valles Centrales del estado de Oaxaca, México. Se articula en datos referidos de los profesores de lengua extranjera de este nivel educativo, al implementar los programas digitales en la formación de docentes. Así mismo, se incluyen las opiniones de los alumnos que cursan la asignatura, para advertir si éstos consideran que los recursos digitales favorecen sus propios procesos de aprendizaje de este idioma. Se determina en la existencia de vinculación entre la Educación y los recursos digitales, para advertir si éstos consideran que los recursos digitales favorecen sus propios procesos de aprendizaje de este idioma. El enfoque metodológico que se siguió fue la encuesta, se determina en la existencia de vinculación entre la Educación y los recursos digitales, que constituyen una práctica de formación integral del estudiante a través de una metodología de enseñanza innovadora del lenguaje, que permita la reflexión y enriquecimiento al relacionarse con el conocimiento, propendiendo así por una educación de calidad.

IMPACTO DEL PROGRAMA JÓVENES EN ACCIÓN EN LA FORMACION INICIAL DE EDUCADORES EN MATEMATICAS. Artículo de investigación elaborado por los académicos Luz Silvana Maldonado Carvajal, Carlos Sebastián Gómez Vergel, Mawency Vergel Ortega, Universidad Francisco de Paula Santander. Examina el impacto del programa Jóvenes en Acción en la permanencia y rendimiento académico de los beneficiarios y no beneficiarios de la Licenciatura en Matemáticas 


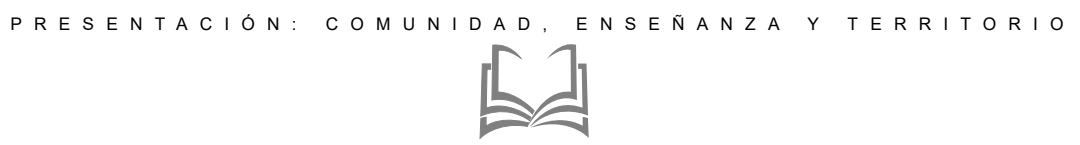

de la Universidad Francisco de Paula Santander. Se sustenta investigaciones sobre la permanencia y el rendimiento académico. Metodológicamente es un estudio con enfoque cualitativo con apoyo del análisis cuantitativo correlacional. Los resultados demuestran un impacto positivo en los estudiantes beneficiarios del programa jóvenes en acción. Se concluye que el apoyo familiar, los procesos de selección a través de pruebas saber y las ayudas económicas contribuyen con la permanencia estudiantil y al rendimiento académico en los estudiantes.

\section{AUTONOMOUS LEARNING OF ENGLISH AS} A FOREIGN LANGUAGE IN A CULTURALLY INTEGRATED B-LEARNING ECOSYSTEM.

Artículo de investigación elaborado por los académicos Lorena Julieth Rojas Salazar:Luis Facundo Maldonado G., Ph.D. Universidad Pedagógica y Tecnológica de Colombia. The low level of oral skills in learning English as a foreign language seems to be related to the lack of spaces and opportunities to interact in dynamic learning environments since the texts and study materials are not related to the context in which the student lives. This research answers the question of whether a b-learning ecosystem with devices for monitoring learning and integrated into cultural dimensions of the students' environment improves the learning of oral skills in learning English. The proposal is based on advances in research on ecosystems of learning and embodied cognition. A system is designed from the specification of learning spaces integrated into a spiral structure. An online learning environment integrates with an ecosystem with elements of Boyacá cuisine to develop communicative interactions and autonomous learning activities. The proposal is validated by taking as population, grade 11 students from the Colombian system and two equivalent samples of selected students, based on previous performance in the English subject in the current school year. Statistical analysis of results supports the positive answer to the research question and supports the importance of the cultural integration of learning $b$ - learning ecosystems in foreign language learning.

\section{MANIFESTACIONES DE LA DINÁMICA FAMILIAR EN FACTORES DE RIESGO SOCIOCULTURALES QUE INCIDEN EN EL CONSUMO DE ALCOHOL EN JÓVENES DE 12 A 18 AÑOS EN TIEMPO DE COVID-19. Artículo} de investigación autoría de las académicas Clara Judith Brito Carrillo, Carmenza Leonor Mendoza Cataño y Angélica María Suarez Brito, Universidad de La Guajira. Dirigido a identificar los factores de riesgo socioculturales que inciden en el consumo de alcohol en jóvenes de 12 a 18 años de edad en la institución educativa Pablo VI en el municipio de Barrancas La Guajira. Los resultados evidencian situaciones problematizadas que requieren de acciones articuladas para buscar alternativas dada la relevancia para la salud mental, así mismo, las bases teóricas, garantizaron el fortalecimiento integrados del objetivo de estudio.

EL MÉTODO "MAYA, artículo de reflexión propositiva elaborado por el académico Jose Henry Maya Ruiz, Universidad e San Buenaventura. Llama la atención sobre la necesidad de disminuir la posibilidad de no naturalizar el conocimiento memorístico sobre el aprendizaje de las tablas de multiplicar. El método "Maya" insiste en aplicar ciertas propiedades de la multiplicación de números naturales y otras estrategias metodológicas, permitiendo que, al manejar el algoritmo de la multiplicación, basta con conocer "de memoria" el producto de 15 números naturales por los dígitos no nulos, haciendo más atractivo el aprendizaje de las llamadas tablas de multiplicar. La utilidad de este método estriba en que es la puerta de entrada al cálculo mental y al desarrollo de habilidades y competencias en matemáticas. 